\section{Population size bottleneck}

SiR-Maynard Smith recently estimated the average human population size since the time of the mitochondrial "Eve". He pointed out that the observed rather small divergence among human mitochondrial

LOW AND HIGH ESTIMATES OF DIVERGENCE ATTAINABLE BY VARIOUS POPULATION SIZES

$$
2 \times 10^{-8} \begin{array}{cc}
\text { Mutation rate per nucleotide per year } \\
10^{-8}
\end{array}
$$

Population size

$\begin{array}{lc}2,000 & 0.0016-0.0064 \\ 3,000 & 0.0024-0.0096 \\ 5,000 & 0.004-0.016\end{array}$

8,000

10,000

20,000

Range of population sizes $2,000-5,000$ have attained any size and still have accumulated mitochondrial diversity at the same rate.

The risk posed to the survival of the population in the first case is equivalent to the risk of crossing Niagara Falls on a tightrope. In the second case, the risk is more like that posed by balancing for a moment on the sharp end of an ocean liner

The three estimates above are based on the values of mutation rate usually cited in the literature ${ }^{3,8}$. The range of population sizes is the range of sizes of the female population that could have given rise to a divergence of $0.57 \%$ for each mutation rate.

genomes of about 0.3 to $0.57 \%$ per nucleotide $^{2.3}$ implies a small average population size of about 5,000 females. Were the average population size any larger, the accumulated divergence would be greater and the "coalescence time", or time back to a single mitochondrial-type, would be correspondingly longer.

If the amount of divergence is known accurately, so is the coalescence time $e^{5,6}$. There is much more uncertainty about the average size of the population leading to a given divergence? ${ }^{7}$ The table shows that the population could easily have been smaller than Maynard Smith suggests. Such small sizes would have to be maintained for thousands of generations, with an attendant high risk of extinction.

A second and more likely possibility is that there was a pronounced bottleneck in numbers at about the coalescence time. After this, the human population could

\section{Trouton's rule}

SIR-John Maddox' pokes gentle fun at chemists, stating that they "are masters at elevating empirical generalizations into natural laws". He cites Trouton's rule as a classical example of this practice. However, I have recently published a simple statistical mechanical rationale of this rule $^{2}$, after one expert in the field admitted $^{3}$ that a satisfactory explanation had never been given, such as that achieved by Einstein for the parallel Dulong and Petit's law of heat capacities of solids. My explanation illuminates the continuity in partition functions in describing changes of state from ideal molecular solids to corresponding ideal liquids to ideal gases. Not only does it reveal the connection with the Einstein heat-capacity theory, but it also shows that the original Eyring transition-state and then proceeding to the blunt end along the promenade deck. It may be that the observed human mitochondrial coalescence time is more than just a statistical accident, and signals a dramatic event in the history of our species.

Department of Biology, CHRISTOPHER WILLS

University of California, San Diego,

La Jolla,

California 92093, USA

1. Maynard Smith, J. Nature 344, 591-2 (1990)

2. Brown. W. M. Proc. natn. Acad. Sci. U.S.A. 77, 36053609 (1980)

3. Cann, R. L., Stoneking, M. \& Wilson, A. C. Nature 325, 31 36 (1987)

4. Kingman, J. F. C. J. appl. Prob. 19A, 27-43 (1982)

5. Nei, M. \& Li, W.-H. Proc. natn. Acad. Sci. U.S.A. 76, 5269 $5273(1979)$

6. Wills, C. in Molecular Genetic Medicine (ed. Friedmann T.) (Academic, New York, in the press).

Avise, J. C., Neigel, J. E. \& Arnold, J. J. molec. Evol. 20 Avise, J. C., Neige

8. Nei, M. in Population Genetics and Molecular Evolution (eds Aoki, K. \& Ohta, T.) 41-64 (Springer, Berlin, 1985).

theory for kinetics is immediately relevant in handling the change from liquid to vapour.

The best analytical form ${ }^{4}$ of Trouton's rule is given by the expression $\Delta S_{b}^{\circ}=R(4+$ $\left.\ln T_{b}\right)$, where $\Delta S_{b}^{\circ}$ is the molar entropy involved, and $T_{\iota}$ is the boiling point. This equation is very readily derived using the following molar partition functions for ideal systems.

\section{Solid (Einstein) $(k T / h v)^{3 *}$}

2. Liquid (cell theory)

$$
(k t / h v)^{3 N} \times e^{v}
$$

3. Gas

$\left(2 \pi m k T / h^{2}\right)^{3 N / 2} \times V^{N} / N !$

4. Liquid/gas transition state $\left(2 \pi m k T / h^{2}\right)^{3 N / 2} \times\left(N \delta^{3}\right)^{N} / N$ !
Here, $k$ is the Boltzmann constant, $h$ Planck's constant, $m$ the molecular mass, $N$ Avogadro's number, $V$ the molar gas volume, and $\delta$ a characteristic transition state distance.

The equivalence of functions 2 and 4 is realized, following Eyring, by substituting the equality $\delta=1 / v(k T / 2 \pi m)^{1 / 2}$, where $v$ is the liquid/gas transition-state frequency.

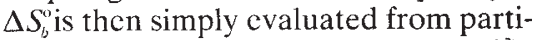
tion functions $2=4$, and 3 as $R 1 n\left(V / N \delta^{3}\right)$, which can be expressed as

$$
R \ln \left(22,410 \times T_{b} / 1.5 \times 273\right)
$$

assuming that $N \delta^{3}$ is a characteristic volume independent of $T$ with a value of $1.5 \mathrm{~cm}^{3}$. This volume is the maximum free molar volume of the ideal liquid in the transition state at its boiling point, so the main result emerging is a specification of the minimum separation (characteristic distance) at which the physical forces between the molecules approximate to zero.

J.J. ROONEY

School of Chemistry,

Queen's University of Belfast,

Belfast BT9 5AG, Northern Ireland

1. Maddox, J. Nature 345, 119 (1990)

2. Rooney, J.J. Proc. R. Ir. Acad. 88B, 39-43 (1988)

3. Nash, L.K. J chem. Ed. 61, 981-984 (1984).

4. Everett, D.H. J.chem. Soc. $2566-2573(1960)$

\section{More pebbles}

SIR-Lorang and Komar pointed out in Scientific Correspondence' that abrasion forces unevenly affect the surface of seashore pebbles. If the force comes evenly from any direction, what will be the resultant shape of the pebbles?

I have some 'experimental' evidence, obtained from my hobby of china-making. When kaolin is manufactured, local rocks, crushed in a 'ball mill', are transformed into clay materials. Rocks with enough water are agitated with hard 'balls', which are natural, round-shaped silicated rocks (quartzite). Abrasion gradually acts on the balls.

I noticed that well-used rock balls had three main configurations: flattened ovoid, ovoid and spherical, in the ratio 70 , 10 and 20 per cent, respectively. Artificial hard 'balls', made from alumina or magnetic iron, are often used instead of the natural balls. These artificial balls keep their original spherical shape even after repetitive usage, although they decrease in size. Thus, I believe that the original structure of the rock material is important for pebble shape, as pointed out by Ashcroft ${ }^{2}$.

Department of Biology,

TOHRU YAZAWA

Tokyo Metropolitan University,

2-1-1 Fukazawa, Setagaya-ku,

Tokyo 158, Japan

1. Lorang. M.S. \& Komar, P.D. Nature 347, 433 (1990)

2. Ashcroft, W. Nature 346, 227 (1990). 